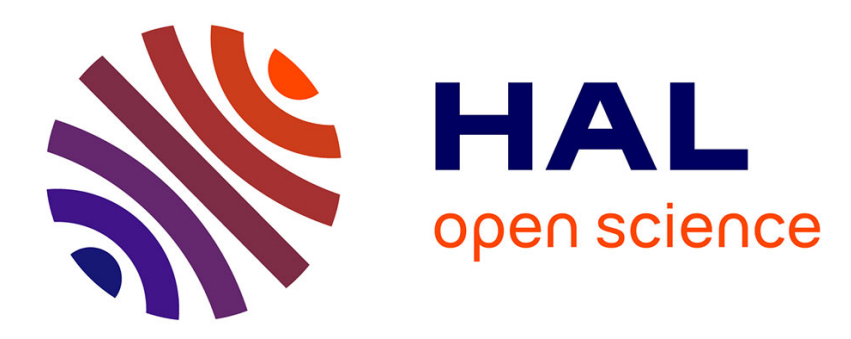

\title{
La coopétition technologique : pourquoi et comment partager sa technologie avec son concurrent ? \\ Sea Matilda Bez
}

\section{To cite this version:}

Sea Matilda Bez. La coopétition technologique : pourquoi et comment partager sa technologie avec son concurrent ?. Innovations - Revue d'économie et de management de l'innovation, 2018, 55 (1), pp.89. 10.3917/inno.pr1.0027 . hal-02466000

\section{HAL Id: hal-02466000 https://hal.science/hal-02466000}

Submitted on 12 Feb 2020

HAL is a multi-disciplinary open access archive for the deposit and dissemination of scientific research documents, whether they are published or not. The documents may come from teaching and research institutions in France or abroad, or from public or private research centers.
L'archive ouverte pluridisciplinaire HAL, est destinée au dépôt et à la diffusion de documents scientifiques de niveau recherche, publiés ou non, émanant des établissements d'enseignement et de recherche français ou étrangers, des laboratoires publics ou privés. 


\section{LA COOPÉTITION TECHNOLOGIQUE : POURQUOI ET COMMENT PARTAGER SA TECHNOLOGIE AVEC SON CONCURRENT?}

\section{RÉSUMÉ}

Partager sa technologie dans un projet impliquant un concurrent est contre-intuitif. En effet, le concurrent va pouvoir internaliser la technologie et la réutiliser contre l'entreprise qui l'a initialement partagée. Alors qu'il existe des structures projets réputées plus sûres car elles permettent de réduire drastiquement le risque d'internalisation des technologies, certaines entreprises préfèrent avoir recours à des structures projets plus risquées qui favorisent l'internalisation. Notre étude explore ce comportement intriguant à l'aide d'une étude de cas approfondie et longitudinale d'une entreprise qui a fait le choix de passer d'une simple structure projet, réputée plus «sûre », à des structures parfois «plus risquées ». Nos résultats montrent non seulement que ce choix est stratégique, mais qu'il existe de nombreuses variables encourageant cette structure plus risquée. Ce changement de structure de projet révèle un changement de représentation des relations de marché entre les concurrents.

Mots-clés : coopétition, partage de technologie, stratégie d'innovation, structure projet, internalisation des technologies

Code JEL: L. Industrial Organization (L1- Firm Strategy) 


\title{
TECHNOLOGICAL COOPETITION: WHY AND HOW TECHNOLOGY WITH A COMPETITOR?
}

\begin{abstract}
Sharing technology on a project involving a competitor is counterintuitive. Indeed, the competitor will be able to internalize the technology and use it against the company who initially shared it. While a project design which drastically reduces the risk of internalization of technology exists, some companies prefer to use riskier project designs that promote internalization. Our study explores this intriguing behavior using an in-depth and longitudinal case study from a company that has made a choice to move from only project design deemed "safer," to sometimes using "riskier". Our results show that this choice is strategic and many variables are encouraging this riskier structure. The change of project design reveals a change in the representation of the market relationship between competitors.
\end{abstract}

Keywords: coopetition, technology sharing, innovation strategy, project design, internalization

Code JEL : L. Industrial Organization (L1 - Firm Strategy) 


\section{LA COOPETITION TECHNOLOGIQUE : POURQUOI ET COMMENT PARTAGER SA TECHNOLOGIE AVEC SON CONCURRENT?}

\section{INTRODUCTION}

La coopération entre entreprises concurrentes pour développer une nouvelle technologie est une stratégie de plus en plus courante (Gnyawali, Park, 2011). On retrouve cette stratégie de coopétition technologique dans des domaines variés allant des semi-conducteurs (Browning, et al., 1995) à la télécommunication (Yami, Nemeh, 2014), en passant par l'aérospatiale (Fernandez, Le Roy, 2015) et la pharmaceutique (Bez et al., 2014). Les recherches antérieures ont permis le développement des connaissances sur la coopétition technologique (e.g., Fernandez, Chiambaretto, 2016; Gnyawali, Park, 2011; Le Roy, Fernandez, 2015; Park, Russo, 1996; Ritala et al., 2015). Ces recherches mettent en évidence les difficultés liées au partage de technologie entre concurrents. En effet, partager sa technologie avec un concurrent signifie la rendre accessible et potentiellement réutilisable par ce concurrent (Park, Russo, 1996). Par suite, les coopétiteurs doivent arbitrer entre le fait de partager pleinement leurs technologies pour augmenter les possibilités d'innovation, et le fait de limiter ce partage des technologies pour contrôler le risque de voir ces technologies réutilisées par un concurrent (Estrada et al., 2016; Ritala et al., 2015). Toute la question est donc de déterminer pourquoi et comment partager la technologie entre concurrents.

Dans ce questionnement, la littérature a identifié deux structures de projet pour manager la coopétition technologique : l'équipe projet séparée et l'équipe projet coopétitive (Fernandez et $a l ., 2017)$. Ces deux structures de projet font référence à deux manières différentes de faire de la coopétition technologique. En effet, même si elles s'accordent sur le besoin d'utiliser et de combiner les technologies stratégiques des concurrents, elles s'opposent sur la nécessité de partager la technologie (i.e., mettre ou non des barrières organisationnelles à l'accès et à la réutilisation de la technologie). Il y a donc un débat sur la nécessité de partager la technologie (Fernandez et al., 2017).

En résumé, la controverse sur les déterminants et les pratiques du partage de technologie est loin d'être close. Cette recherche se propose de contribuer à ce débat en étudiant de façon approfondie un cas de coopétition technologique. L'étude de cas choisie porte sur les projets d'exploration et de production (E\&P) de pétrole et de gaz de l'entreprise Total.

Cette recherche contribue à la connaissance sur trois points. Premièrement, elle confirme l'existence de deux managements différents en matière de partage de technologie (i.e., l'équipe projet séparée et l'équipe projet coopétitive). Deuxièmement, elle montre que les déterminants du partage de la technologie entre concurrents sont nombreux et multiniveaux. In fine, cette recherche identifie une nouvelle typologie de la coopétition distinguant la coopétition basique de la coopétition avancée. Cette nouvelle typologie complète les typologies existantes et améliore la compréhension de la coopétition.

\section{LA COOPÉTITION TECHNOLOGIQUE ET LE PARTAGE DE LA TECHNOLOGIE STRATÉGIQUE}

La coopétition est avant tout une révolution cognitive dans laquelle la coopération et la compétition peuvent se produire simultanément entre des acteurs, qui deviennent des partenaires-adversaires (Brandenburger, Nalebuff, 1996; Gnyawali et al., 2016). Cette simultanéité de la compétition et de la coopération est le fondement même de la notion de coopétition. Elle est à la base d'un avantage concurrentiel (Quintana-García, Benavides- 
Velasco, 2004; Ritala, Hurmelinna-Laukkanen, 2009). En effet, la combinaison des capacités et des ressources de concurrents directs génère plus de valeur qu'une entreprise seule ou qu'une entreprise avec d'autres partenaires non-concurrents (Bouncken, Fredrich, 2012; Gnyawali, Park, 2011). Les recherches antérieures ont mis en avant la variété des activités pouvant concerner la coopétition : allant de la R\&D à l'innovation technologique, en passant par la logistique, la production, voire même la vente (Bengtsson, Kock, 2000; PellegrinBoucher et al., 2013). Un des résultats issu de cette littérature est le rôle clé de la coopétition dans le développement de nouvelles technologies (Bouncken, à paraitre; Gnyawali, Park, 2011). Le développement d'une nouvelle technologie est un processus coûteux, incertain et long. De plus, la nouvelle technologie développée peut être rapidement remplacée par une autre technologie encore plus performante avant même que les investissements ne soient rentabilisés. Face à ces défis, partager et combiner ses technologies entre concurrents est une solution pour gagner en rapidité de mise sur le marché, réduire le coût de développement ainsi que le risque de développement (Ritala, Hurmelinna-Laukkanen, 2009). Dit autrement, partager ses technologies maximise les chances de succès du développement d'une nouvelle technologie. En utilisant une approche par les ressources, les recherches antérieures vont même plus loin et soutiennent que le partage doit impliquer des ressources stratégiques pour profiter de la valeur créée par la présence simultanée de coopération et de compétition (Gnyawali, Charleton, 2017). Une entreprise ne devient attractive pour un projet de coopétition technologique qu'à partir du moment où elle possède et accepte de partager ses technologies qui lui génèrent des rentes (Fernandez, Chiambaretto, 2016; Gnyawali, Charleton, 2017). De plus, la valeur capturée dans une stratégie de coopétition technologique dépend directement de la valeur créée et donc des ressources stratégiques apportées initialement. Ainsi, si une entreprise ne partage pas une de ses technologies stratégiques, elle réduit la valeur capturée du projet commun. Par exemple, lorsque les deux concurrents de l'aérospatiale Astrium et TAS se sont alliés pour codévelopper un satellite innovant, le projet n'atteignit pas la performance attendue. Les équipes opérationnelles refusaient de partager les technologies nécessaires pour la cocréation de ce satellite radicalement nouveau. Elles jugeaient ces technologies trop stratégiques pour être partagées. Ce n'est qu'à partir du moment où elles ont réussi à partager les technologies stratégiques que le projet a pu aboutir à un satellite innovant (Fernandez, Le Roy, 2010).

Néanmoins, si ce partage de technologie permet à une entreprise de capturer de la valeur et même de développer un avantage concurrentiel, ne pas partager ses technologies est aussi une stratégie pertinente. En effet, le partage de technologie dans une stratégie de coopétition technologique peut simultanément nuire à l'avantage concurrentiel actuel ou futur de l'entreprise. Bouncken et Kraus (2013) parlent des deux côtés d'une même pièce : il n'est pas possible de bénéficier des avantages du partage de technologie avec un concurrent sans prendre le risque d'une détérioration de son avantage concurrentiel actuel ou futur. Partager sa technologie avec un concurrent implique de prendre le risque de subir un comportement opportuniste du concurrent (Park, Russo, 1996), une fuite de connaissances involontaire (Dyer, Singh, 1998) et une course à l'apprentissage (Hamel, 1991). Par exemple, lors de la coopération entre les deux groupes pharmaceutiques concurrents, Sanofi et Bristol Myers Squibb, ce dernier a utilisé les connaissances et technologies partagées par Sanofi pour tenter de développer un produit concurrent à celui qu'ils développaient ensemble (Bez et al., 2014). Ainsi, avant de s'impliquer dans une relation de coopétition, les entreprises sont confrontées à un paradoxe. Elles peuvent partager leur technologie avec un concurrent pour maximiser la valeur créée, tout en prenant le risque de s'affaiblir à long terme en rendant leur technologie accessible et réutilisable par le concurrent. Ou bien, elles décident de ne pas partager pour ne pas risquer une fuite involontaire de technologie, prenant alors le risque de réduire la valeur potentielle créée. 


\section{LE MANAGEMENT DE LA COOPETITION : PARTAGER OU NE PAS PARTAGER SA TECHNOLOGIE}

Un courant de recherche spécialisé sur le management de la coopétition s'est développé suite aux résultats empiriques ambivalents de l'impact de la coopétition sur le développement de nouvelles technologies (Arranz, de Arroyabe, 2008; Nieto, Santamaría, 2007; Santamaria, Surroca, 2011). Ce courant repose sur l'idée que le management est le lien manquant entre le coopétition et la performance de la stratégie de coopétition (Le Roy, Czakon, 2016). Sans un management adéquat, la relation théorique gagnant-gagnant de la coopétition pourrait devenir une relation gagnant-perdant (Park, Russo, 1996). L'objectif de ce courant de recherche est de comprendre comment maximiser les bénéfices de la coopétition sans partager les technologies stratégiques (i.e., sans prendre le risque de perte dû à l'internalisation des technologies par le concurrent). Baumard (2010, p.93) parle de réussir à «partager sans partager ». Plus concrètement, Fernandez et Chiambaretto (2016) montrent qu'il est possible de monter des parois de protection qui permettent de contribuer au projet avec sa technologie sans prendre le risque de l'internalisation.

Une des contributions de ce courant est d'avoir identifié une solution organisationnelle qui permette d'utiliser simultanément les technologies des concurrents sans prendre le risque de l'internalisation de ces dernières par le concurrent (e.g., Faems, Janssens, Van Looy, 2010; Fernandez et al., 2017; Oxley, Sampson, 2004). Cette dernière consiste à mettre en place une équipe projet séparée (Fernandez et al., 2017) aussi connue sous le nom de structure "plugin » de Hamel (1991). Concrètement, le projet commun est segmenté en tâches ou en modules, puis chaque coopétiteur effectue en interne les tâches qui lui sont attribuées. A aucun moment la technologie n'est partagée. Seul les outcomes de son utilisation le sont. Cette structure limite l'accès à sa technologie par le concurrent tout en contribuant activement au succès du projet. Cette structure est présentée comme la structure optimale contre le risque d'internalisation des connaissances par le concurrent (Faems et al., 2010).

Cependant, les études exploratoires, sur des cas de coopétition réussis, mettent aussi en avant des structures de projet différentes : équipe projet coopétitive (Fernandez et al., 2017). Dans une équipe projet coopétitive, les équipes des deux entreprises concurrentes sont mélangées et travaillent ensemble quotidiennement. Cette organisation implique des interactions intenses et régulières basées sur le partage réciproque de technologies. Cette structure de projet est contre-intuitive car, en augmentant les interactions entre concurrents, elle augmente le risque d'internalisation des technologies par le concurrent.

Cependant, la coexistence de ces deux structures de projet a été confirmée empiriquement par une étude de cas sur les projets de coopération entre les deux concurrents de l'aérospatiale Airbus et Thalès (Fernandez et al., 2017), mais aussi dans l'industrie de la défense décrite par Depeyre, Dumez (2007). En effet, une relecture de l'étude de cas de Depeyre, Dumez (2007) ${ }^{1}$ sous l'angle du partage de technologies permet d'identifier deux partages différents de technologie. La première forme consiste à ne pas partager les technologies. La coopération entre concurrents se base sur des concurrents fournissant des composants bien spécifiques (i.e., premières formes de coopétition datant des années 1993 à 2000). Par la suite, face à un projet complexe impliquant une technologie tellement nouvelle, une deuxième forme de coopétition basée sur le partage de technologie a été identifiée. Cette dernière recherche

\footnotetext{
${ }^{1}$ L'étude de cas de Depeyre, Dumez (2007) n'avait pas pour objectif d'étudier le partage de technologie. Ils analysaient les interactions stratégiques entre client et fournisseur afin de mettre en avant le rôle du client dans le développement du phénomène de coopétition.
} 
l'interaction intense et le partage de technologie entre les deux équipes. L'interaction est tellement importante que les «deux firmes vont apprendre l'une de l'autre au cours de leur travail commun et chacune va pouvoir, au long de cet apprentissage, compléter en dynamique ses capacités avec celles qui lui manquaient » (Depeyre, Dumez, 2007, p. 108). Cette dernière étude de cas illustre bien les enjeux différents des stratégies de coopétition technologique basées sur le partage ou l'absence de partage de technologie. Si les entreprises font le choix de partager leurs technologies, elles favorisent le développement d'une technologie totalement nouvelle, mais elles prennent le risque de renforcer le concurrent. Fernandez et al. (2014) parlent d'armer son concurrent avec ses propres armes.

Jusqu'à présent, peu de recherches en coopétition ont exploré les déterminants du partage, ou non, de technologie dans les relations de coopétition technologique (à l'exception de certains travaux comme Fernandez et al., 2017). La littérature sur le management de la coopétition s'est principalement attachée à discuter la capacité ou non des individus à intégrer le paradoxe et à en déduire des principes de management. A ce stade, il devient intéressant de comprendre quels sont les déterminants de ce partage ou non de technologie.

\section{L'ÉTUDE QUALITATIVE}

L'objectif de cette recherche est de mieux comprendre les déterminants du partage ou du non partage de technologie dans les stratégies de coopétition technologique. Afin d'identifier les déterminants et d'avoir une compréhension dynamique de ces différentes modalités de partage, nous avons étudié les stratégies de coopétition technologique dans les activités d'exploration et production $(\mathrm{E} \& \mathrm{P})$ du groupe pétrolier Total, de sa création à aujourd'hui. L'étude de cas est la méthode recommandée quand l'objectif est de comprendre les aspects dynamiques et paradoxaux de la coopétition (Fernandez, Chiambaretto, 2016; Gnyawali, Park, 2011). Une approche longitudinale permet une compréhension holistique du phénomène à étudier (Dumez, 2013; Eisenhardt, 1989; Yin, 2014).

L'étude de cas choisie porte sur les projets d'E\&P de pétrole et de gaz de l'entreprise Total. Total compte plus de soixante-dix années d'expérience en matière de coopétition technologique. Elle fait donc figure de modèle en matière de coopération réussie entre grands groupes concurrents, nécessitant le développement d'une nouvelle technologie. De plus, dans les années 70, elle devient même emblématique grâce à ses choix stratégiques; de simples structures de projet dites «sûres » (i.e., équipe projet séparée), Total est passée par la suite à des structures parfois plus risquées (i.e., équipe projet commune). Ces deux structures continuent même de coexister au sein de l'organisation Total. De plus, en choisissant d'étudier les activités d'E\&P de pétrole et de gaz d'une entreprise comme Total, nous étudions des projets longs, coûteux, complexes et risqués :

- Longs : la durée moyenne des projets d'E\&P réussis s'étend sur une vingtaine d'années.

- Coûteux : quelle que soit l'issue du projet, les montants investis sont importants. Avant de trouver un réservoir de pétrole potentiellement exploitable, une entreprise fore 2 à 10 puits. Or, un puits sur terre coûte entre 25 et 40 millions d'euros, et un puits off-shore (i.e., un puits haute mer) coûte entre 650 et 900 millions d'euros. Une campagne d'exploration, (i.e., la partie « investissement » en gris foncé sur la figure 1) peut représenter un coût allant de 200 millions d'euros à 50 milliards d'euros. En cas d'échec, le montant investi est une perte sèche.

- Complexes et risqués : car la probabilité d'arrêt du projet avant d'être considéré comme réussi est élevée (cf. figure 1 - les croix matérialisent trois évènements critiques conduisant à l'arrêt du projet). 
Dans des projets longs, coûteux, complexes et risqués, la technologie joue, par définition, un rôle clé dans l'avantage concurrentiel de l'entreprise.

Figure 1-L'activité d'E\&P : un processus long, coûteux et risqué

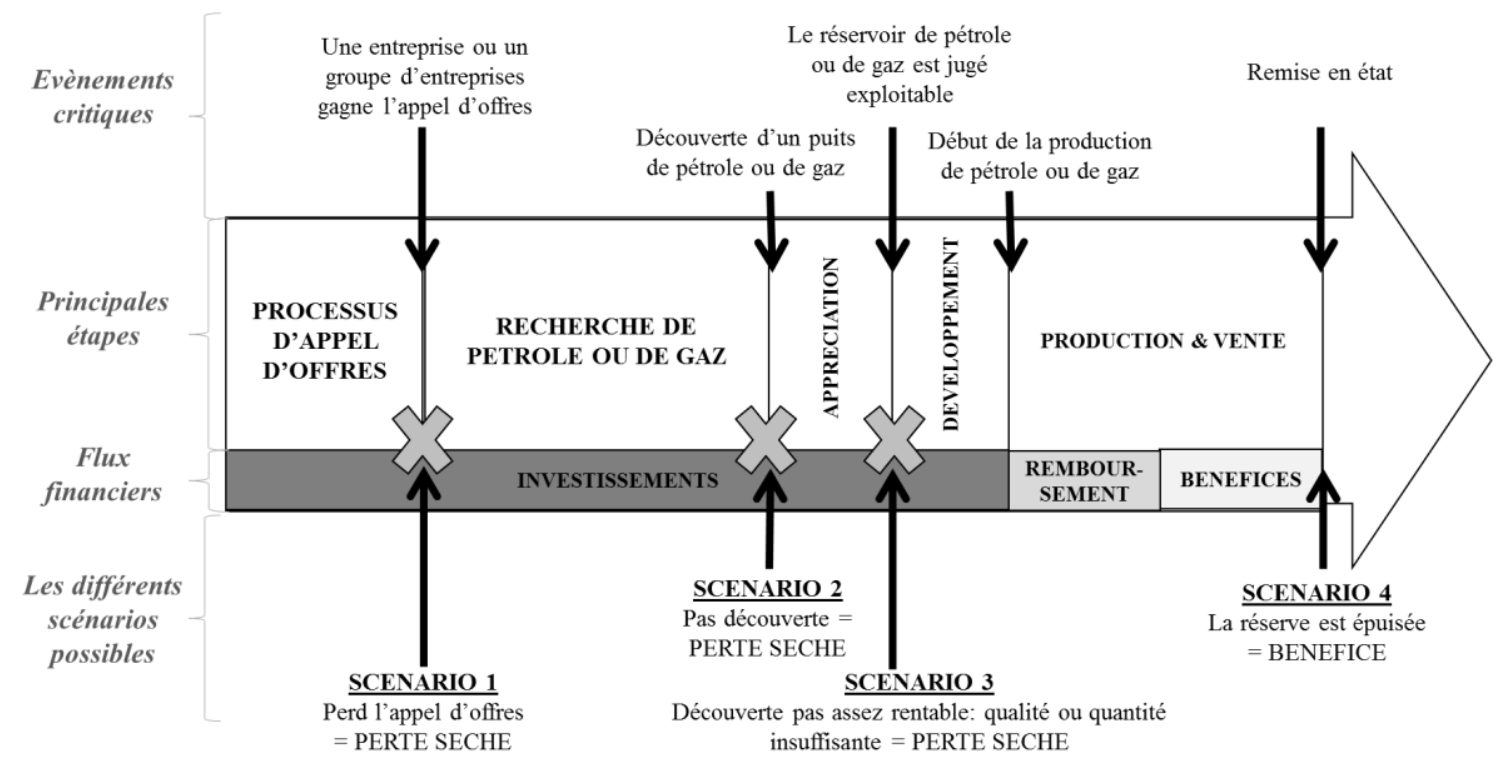

Source : auteur

Notre étude de cas repose sur une collecte de données secondaires et primaires. Ces derniers ont permis de créer un résumé narratif des stratégies d'E\&P de Total, de sa création en 1920 jusqu'à aujourd'hui (cf. tableau 2). Pour s'assurer de la validité interne de nos données, chaque évènement critique ou modalité de partage identifié, a été triangulé par des sources différentes.

Dans le but de ne pas biaiser ce dernier, nous avons constitué le résumé narratif avant de définir la grille d'analyse de cet article (Dumez, 2016). La grille d'analyse utilisée est une grille inspirée de la carte conceptuelle de Dorn et al. (2016). En effet, à partir d'une synthèse systématique de la littérature de la coopétition, les auteurs ont identifié 4 niveaux pouvant impacter les pratiques de management de la coopétition : (1) la nature de la relation, (2) les outputs de la relation, (3) les caractéristiques des acteurs et, (4) les caractéristiques de l'environnement. 


\begin{tabular}{|c|c|}
\hline $\begin{array}{l}\text { Nature des } \\
\text { données }\end{array}$ & Détails \\
\hline Entretiens & $\begin{array}{l}\text { Nombre : Vingt personnes (dont une avec laquelle nous avons fait } \\
\text { plusieurs entretiens). } \\
\text { Modalité de collecte : Eentretiens semi-directifs réalisés en face-face ou } \\
\text { par téléphone. } \\
\text { Durée moyenne : } 50 \text { minutes (à l'exception d'une des personnes avec } \\
\text { laquelle plusieurs entretiens ont été effectués pour une durée totale de } \\
\text { 9h). } \\
\text { Trois niveaux de responsabilité : } \\
\text { - } \quad \text { quatre entretiens avec les hauts dirigeants, au sein de la maison } \\
\text { mère de Total, qui interviennent dans les décisions de } \\
\text { coopération dans les projets d'exploration et production au } \\
\text { niveau global ; } \\
\text { sept entretiens avec des managers d'actifs spécifiques, qui ont } \\
\text { une vision globale et une influence sur les décisions au niveau } \\
\text { de l'ensemble des projets avec un partenaire dans une zone } \\
\text { géographique; } \\
\text { neuf entretiens avec des opérationnels qui participent aux } \\
\text { projets d'exploration et production avec des partenaires. }\end{array}$ \\
\hline De l'observation & $\begin{array}{l}\text { Deux jours d'immersion dans une formation au sein de Total concernant } \\
\text { notre objet de recherche, en l'occurrence les consortiums avec des } \\
\text { partenaires externes dont les concurrents. }\end{array}$ \\
\hline $\begin{array}{l}\text { Livre publié et } \\
\text { articles } \\
\text { académiques }\end{array}$ & $\begin{array}{l}\text { Livre de l'association internationale des négociateurs de pétrole écrit par } \\
\text { Walker en } 2010 \text {, } \\
\text { Livre « The Petroleum Handbook» publié en } 1986 \text { par Shell. }\end{array}$ \\
\hline $\begin{array}{c}\text { Les rapports } \\
\text { annuels }\end{array}$ & Rapports annuels de Total de 2002 à 2016. \\
\hline $\begin{array}{l}\text { Articles de presse } \\
\text { et base de données } \\
\text { de Total }\end{array}$ & $\begin{array}{l}\text { Pour approfondir nos connaissances sur les éléments issus des entretiens } \\
\text { ou des données secondaires: } \\
\text { - } \\
\text { - } \\
\text { Des articles de l'actualité de Total entre } 2002 \text { et } 2016, \\
\text { La base de données virtuelles de Total constituéeà partir } \\
\text { d'archives du Groupe Total (disponible sur internet). }\end{array}$ \\
\hline
\end{tabular}

Source : auteur

\section{LA TECHNOLOGIE COMME NERF DE LA GUERRE}

Dans les activités d'exploration et production de pétrole et de gaz, Total est une entreprise pétrolière en concurrence avec les autres entreprises pétrolières comme Exxon Mobil, Shell, Chevron, BP ou ConocoPhillip. Dans leur rivalité, la technologie est le nerf de la guerre. L'avantage concurrentiel généré par la possession d'une technologie de pointe est triple : (1) diminution du risque et des investissements nécessaires dans un projet d'exploration et production (E\&P); (2) augmentation des chances de gagner un appel d'offres; (3) augmentation des opportunités de participer à des projets d'exploration et production sans avoir gagné l'appel d'offres (cf. figure 2). 
Figure 2 - Les déterminants des rentes économiques de l'entreprise Total

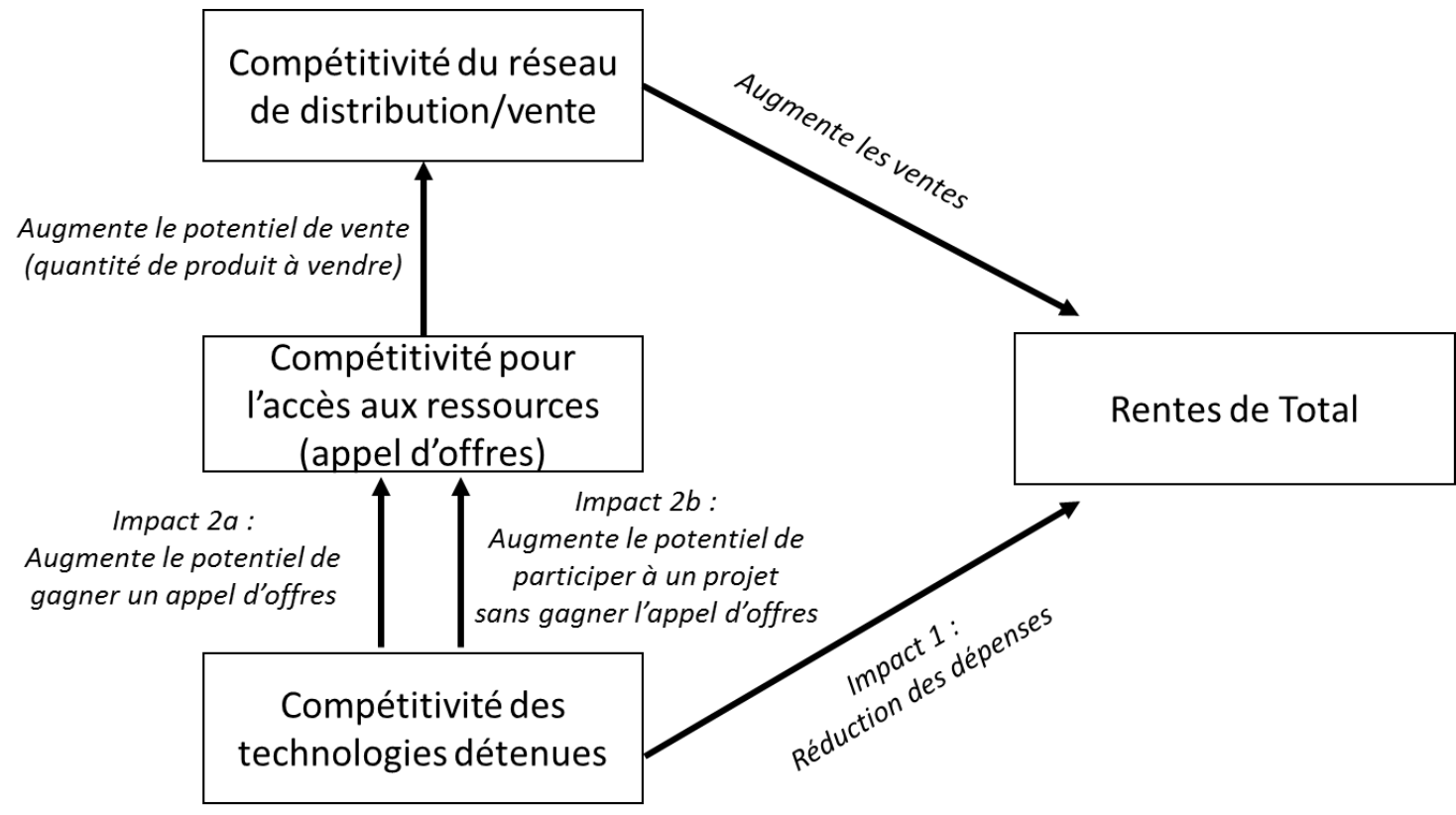

Source : auteur

Par exemple, Total possède une technologie stratégique permettant de modéliser avec plus de précision les sous-sols pour identifier de potentiels réservoirs de pétrole. Pouvoir modéliser avec plus de précision les sous-sols permet de réduire le nombre de puits forés avant la découverte d'un réservoir. Cette technologie est donc stratégique car elle augmente les chances de Total de trouver un réservoir de pétrole rapidement et réduit le niveau d'investissement nécessaire. Cette capacité à augmenter les chances de succès et de réduire les coûts va attirer les Etats et donc augmenter les chances de l'entreprise de gagner les appels d'offres. De plus, même les concurrents peuvent être intéressés et vouloir utiliser la technologie de l'entreprise dans les projets où elle n'est pas présente. Ils proposeront donc à l'entreprise de devenir actionnaire dans le projet et d'apporter sa technologie. Ainsi, avoir une technologie stratégique augmente la probabilité de créer de la valeur dans un projet mais aussi de participer à des projets desquels ils auraient été exclus.

La technologie est bien le nerf de la guerre pour une entreprise comme Total. Développer de nouvelles technologies permet d'avoir un avantage concurrentiel et de générer des rentes dans les projets d'E\&P.

\section{L'ÉVOLUTION DES STRATÉGIES DE COOPÉRATION DE TOTAL AVEC SES CONCURRENTS}

Des années 20 au milieu des années 50, Total n'a pas de stratégie de coopétition technologique. Mais cette période est clé car elle permet à Total de développer sa première technologie stratégique. Ce n'est qu'à partir du milieu des années 50 que Total commence à effectuer des stratégies de coopétition technologique: Total commence à combiner ses technologies avec celles de ses concurrents. Cette combinaison assure l'efficience technologique de leurs projets d'E\&P. Puis dans les années 70 , en parallèle à cette première coopétition technologique, Total dépasse la combinaison de technologies pour mettre en place un processus de cocréation de technologies avec ses concurrents. Dans les zones considérées comme inaccessibles, faute de technologie adéquate, Total coopère avec certains de ses 
concurrents pour cocréer une nouvelle technologie leur permettant ensemble d'explorer et de produire du pétrole dans les zones théoriquement inexploitables (e.g., en haute mer).

Cette section résume de manière historique l'évolution des stratégies de coopération de Total et sera divisée en trois parties pour suivre les trois grandes périodes identifiées (cf. figure 3).

Figure 3 - Evolution des stratégies de coopération avec des concurrents de l'entreprise Total

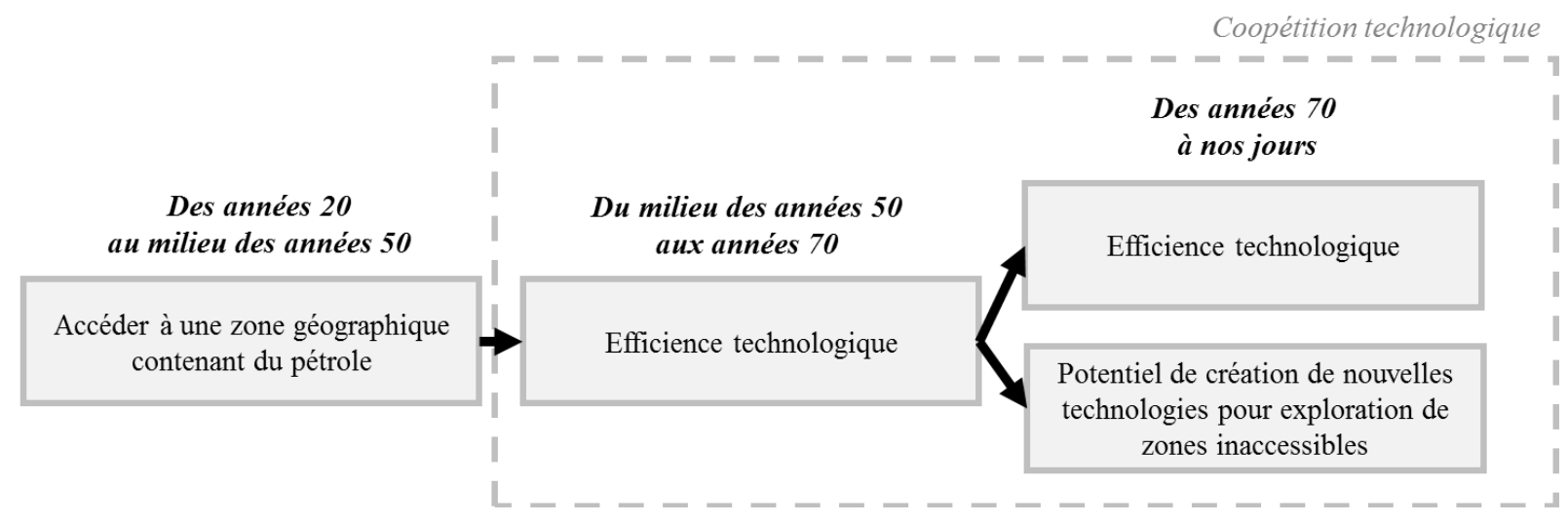

Source : auteur

\section{Les premières formes de coopération et la création d'une technologie stratégique (1920- 1950)}

Dès sa création en 1924, Total coopère avec ses concurrents. Cette coopération n'implique pas le développement de nouvelles technologies mais consiste uniquement à accéder au pétrole produit.

L'entreprise française Total, qui à l'époque s'appelle « la compagnie pétrolière française », se retrouve face à un dilemme : elle veut produire son propre pétrole mais elle ne peut pas accéder à des sols contenant du pétrole, ni en France, ni à l'étranger. En effet, le sol français contient peu de pétrole ${ }^{2}$ et les «majors » de l'époque ${ }^{3}$ détiennent déjà l'ensemble des territoires étrangers connus pour leurs réservoirs de pétrole. Lors d'un de nos entretiens, un haut dirigeant nous a expliqué : "Il n'y avait plus d'espace à conquérir, le territoire était déjà en partie réparti [par les majors].»

Après la première guerre mondiale, l'entreprise qui deviendra Total réussit à entrer dans un projet d'E\&P. En effet, suite aux indemnités de guerre, la banque allemande donne ses parts dans l'entreprise l' «Iraq Petroleum Compagnie » (IPC) ${ }^{4}$. Cette participation est stratégique pour la future entreprise Total $^{5}$ car l'IPC détient le monopole sur l'exploration et la production en Mésopotamie. Cette entreprise sans but lucratif a pour objectif de produire du pétrole pour les entreprises actionnaires. Les entreprises actionnaires sont des entreprises qui s'affrontent farouchement sur le marché mondial du pétrole, mais qui au sein du projet, se partagent les droits de propriété et les décisions stratégiques (cf. figure 4). En 1928, lors de la découverte du premier gisement de pétrole, IPC est détenu à 23,75\% par la «Near East

\footnotetext{
${ }^{2}$ Les recherches de pétrole sur le sol français entre 1920 et 1935 révèlent que ce dernier est pauvre en pétrole à l'exception de quelques petites découvertes et d'un gisement de gaz naturel important mais très difficile techniquement.

${ }^{3}$ ExxonMobil, Shell, Chevron, BP ou ConocoPhillip

${ }^{4}$ A l'époque le nom de IPC était « Turkish Petroleum Company (TPC)».

${ }^{5}$ Plus précisément, c'est suite à la réception de ces parts, que la « compagnie pétrolière française » est créée.
} 
Development Corp. » (NPC) ${ }^{6}$, la Royal Dutch Shell et la future entreprise Total $^{7}$; et les $5 \%$ restant sont détenus par le fondateur. A cette époque, les premières coopérations de Total avec ses concurrents n'impliquent pas de développer une technologie en commun et Total reste uniquement un actionnaire (pas l'opérateur) (cf. figure 4, Total en tant qu'une des entreprises actionnaires est représentée par la couleur gris foncé).

Figure 4 - Les projets de coopération entre concurrents sans partage de technologie

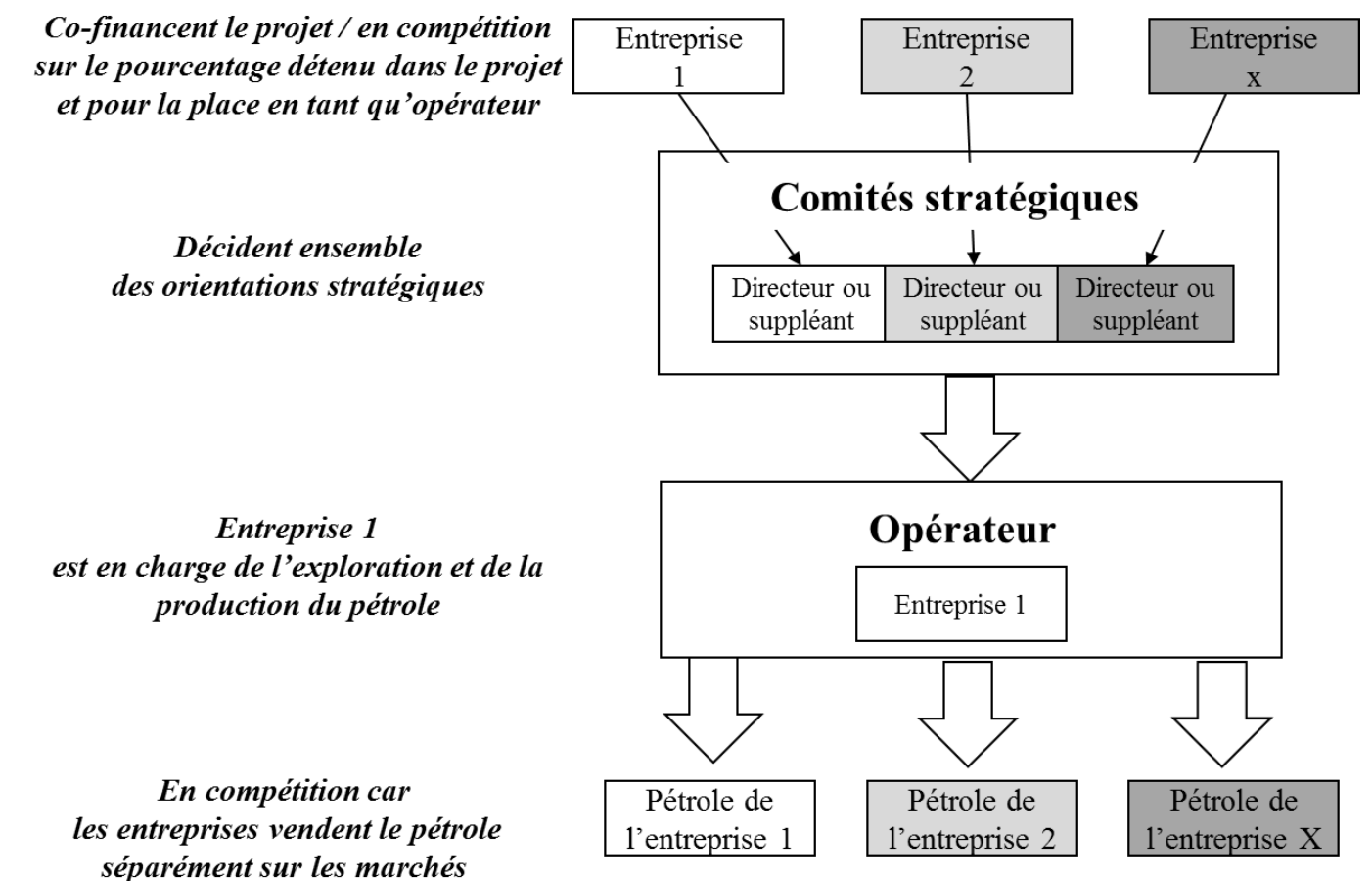

Source : auteur

Puis, l'entreprise qui deviendra Total veut s'émanciper de ses concurrents. Elle décide alors d'explorer seule de nouveaux territoires dont les potentialités en matière de pétrole sont encore inconnues. Son objectif est de pouvoir répondre à la totalité de la demande française et donc d'accroître sa production de pétrole. L'entreprise se tourne alors vers les seuls territoires accessibles pour Total : la France et ses colonies françaises. Sur le sol français, la persévérance paye car en 1941 Total découvre du pétrole dans la commune de Lacq à 600 mètres du sol. Le gisement de pétrole de Lacq est exploité de 1950 à 1955 et produit 1460000 tonnes; il devient le premier gisement français. Mais, au milieu des années 50, le puits fournit de moins en moins de pétrole. Suite à la découverte de ce puits de pétrole, des recherches sont conduites aux alentours et en 1951, du gaz à très forte pression est alors découvert à 3545 mètres. Cependant Total rencontre des problèmes techniques pour descendre à cette profondeur : le gaz dévore les parois des tubes d'acier.

Au regard de la difficulté technologique et se sentant dépassée techniquement, le premier réflexe de l'entreprise Total a été de chercher à coopérer avec ses concurrents qui avaient plus d'expérience. Finalement, Total gère à $100 \%$ ce projet non par choix mais parce que les potentiels partenaires ont refusé de coopérer, jugeant la ressource impossible à exploiter. Total continue à faire des recherches seul et réussit à concevoir, grâce aux ingénieurs

\footnotetext{
${ }^{6}$ Un consortium de cinq entreprises pétrolières américaines.

${ }^{7}$ A l'époque, la Compagnie française des pétroles (CFP).
} 
de Vallourec un acier capable de résister à la corrosion et imagine un outil de désulfuration du gaz. En 1957, l'entreprise réussit à dépasser la contrainte technique et à inaugurer la plus grande usine de gaz d'Europe.

\section{Total combine ses technologies avec celles de ses concurrents (1950-1970)}

Jusqu'aux années 1950, la répartition des zones géographiques contenant du pétrole était figée par des enjeux politiques et historiques liés aux contrats de concessions et l'existence de colonies. A la fin des années 1950, les entreprises internationales prennent conscience de la nécessité de diversifier leur offre de pétrole. En effet, la majorité des entreprises constate qu'augmenter les projets d'E\&P permet de lutter contre la diminution de production dans leur pays d'origine et de réduire leur dépendance à l'OPEC.

L'indépendance des colonies arrive à point nommé, la compétition se libère en partie des biais politiques et historiques. L'accès aux territoires géographiques est donné aux entreprises les plus avantageuses pour le pays, c'est-à-dire aux entreprises capables de maximiser l'exploitation à moindre coût. Une compétition féroce commence pour l'obtention de ces zones géographiques. Dans le livre qui relate l'histoire de l'AIPN, nous apprenons que «les fondateurs de AIPN se sont fait les dents en termes de négociation dans ces pays [les anciennes colonies] » (Walker, 2010).

A cette époque, grâce au projet d'E\&P dans la commune de Lacq, l'entreprise Total a démontré sa capacité à innover et possède désormais une technologie de pointe supérieure à celles des autres majors (i.e., acier capable de résister à la corrosion). Ainsi, Total devient un adversaire de taille et concurrence les majors dans les nouveaux appels d'offres. A plusieurs reprises, Total gagne seul certains appels d'offres.

Cependant, plus la zone géographique contient potentiellement du pétrole facile d'accès, plus la compétition est intense. Or, la compétition au niveau technologique est tellement forte et évolue si vite qu'un investissement intense en R\&D n'est pas suffisant pour maintenir une supériorité technologique. Pour gagner les appels d'offres, l'objectif devient alors de constituer l'offre la plus intéressante pour les Etats en s'associant avec les concurrents possédant des technologies complémentaires. L'offre commune est bien supérieure en technologie à l'offre que Total ou ses partenaires/concurrents auraient pu proposer seule. En cas de gain de l'appel d'offre, toutes les entreprises de l'offre gagnante financent le projet et bénéficient du pétrole. L'entreprise la plus avancée techniquement devient l'opérateur et prend en charge les opérations d'E\&P. L'opérateur est conscient qu'il ne détient pas toutes les meilleures technologies et délègue certaines tâches à d'autres entreprises du projet en fonction de leurs compétences technologiques. Ces dernières effectuent la tâche dans leur entreprise et ensuite partage les résultats (cf. figure 5). Par exemple, l'entreprise Total possède un des ordinateurs les plus puissants du monde. Lorsque l'entreprise Total n'est pas opérateur, l'opérateur lui confie souvent les calculs de modélisation. Total effectue les calculs de modélisation en interne et ne partage que les résultats.

Se voir confier une des tâches est source d'avantage concurrentiel. En effet, si une technologie a été choisie dans un projet, cela devient une preuve que les concurrents valident la supériorité de cette technologie ou de ce savoir-faire. Concrètement, quand Total réussit à être l'opérateur, ou à avoir ses technologies utilisées dans des projets d'E\&P réussis, cela certifie de manière empirique leur bon fonctionnement, et cela affirme leur supériorité. Un des directeurs interviewés soutient : "Dans ce métier être opérateur c'est mieux que de ne pas l'être. C'est une vitrine technologique [qui] permet de se développer, de valoriser nos compétences ». Ainsi, la compétition pour les appels d'offres n'est que la partie émergée de la compétition entre les grands groupes pétroliers. Une compétition existe au sein des consortiums. Dans ces derniers, les entreprises sont en compétition soit pour être l'entreprise 
opératrice, soit pour le choix de la technologie/savoir-faire qui sera utilisée dans le projet. Au regard du risque et du coût financier d'un projet d'E\&P, tous les partenaires ont intérêt à utiliser la meilleure technologie et à confier le projet au meilleur opérateur. Par exemple, en mars 2016, le projet d'exploration et de production commencé en 1990 au Kazakhstan ne produisait toujours pas de pétrole alors que les entreprises avaient investi plus de 50 milliards de dollars (montant cinq fois supérieur au devis initial) ${ }^{8}$.

Figure 5 - Coopération technologique entre concurrents (additionnent la meilleure technologie de chacun des participants)

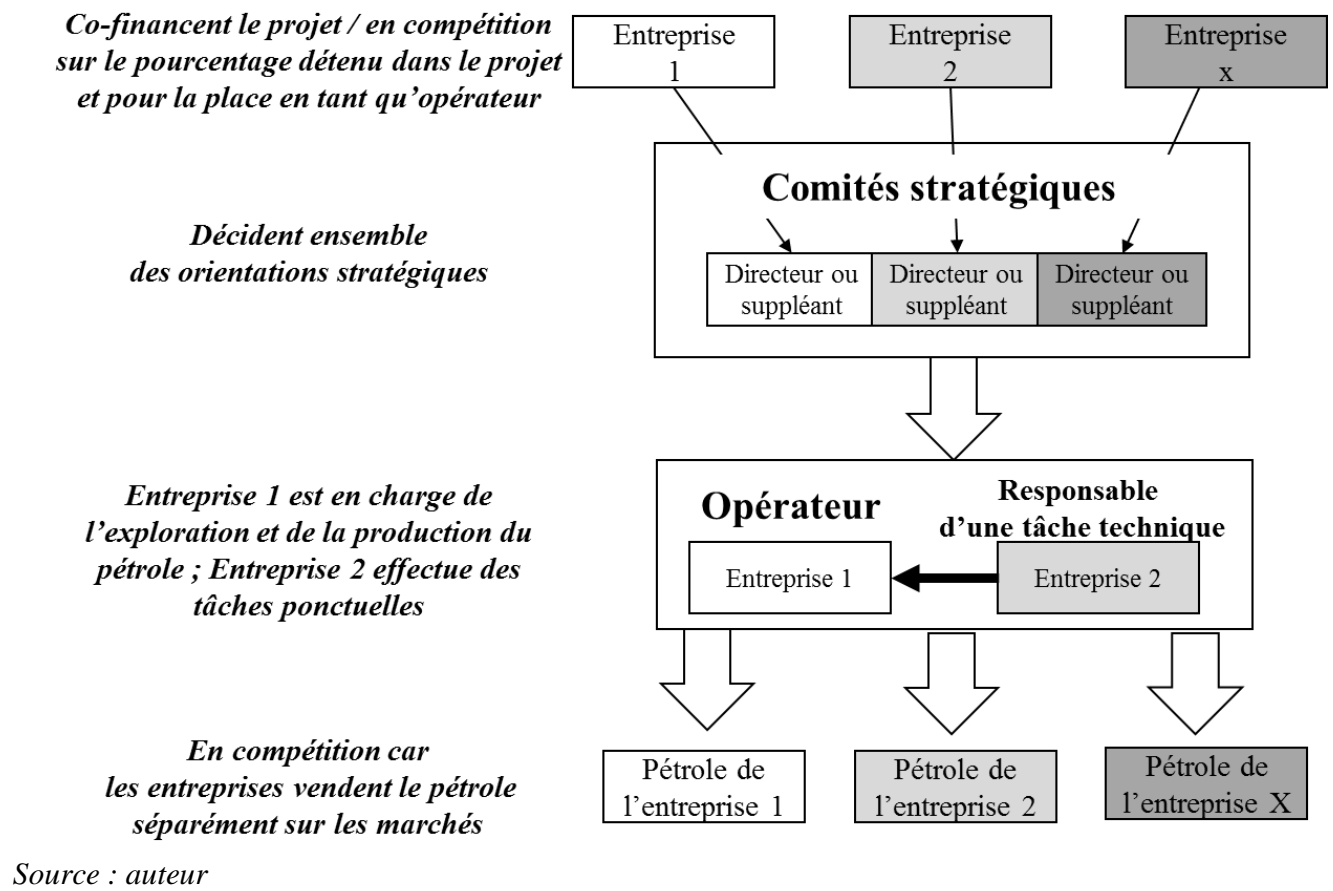

Combiner les technologies avec un concurrent permet donc à Total d'augmenter son attractivité. Cela augmente aussi ses chances d'accéder à de futurs projets d'exploration et production. Si cet avantage est clé, ce n'est pas le seul. La combinaison des technologies a pour premier avantage d'assurer une efficience technologique. En outre, elle augmente la probabilité de succès et de profit du projet. Ainsi, même quand Total a été le seul à percevoir le réel potentiel d'un bloc encore méconnu, et qu'il a réussi à gagner l'appel d'offres seul, il peut proposer aux concurrents battus lors de l'appel d'offres de collaborer. La collaboration est presque considérée comme obligatoire pour optimiser l'exploration et l'exploitation de larges projets complexes. A ce propos, un manager interviewé soutient que : "Mais on a quand même chez Total quelques politiques de champ où l'on est tout seul. Un autre cas de figure, on peut commencer à $100 \%$ TOTAL. Le Comex [dit] OK on peut y aller. Et pendant l'exploration on a des informations et on se dit "oh, là là c'est gros » [et donc on cherche si un autre groupe international aimerait participer]. "Inversement, même si Total a été battu lors de l'appel d'offres, le gagnant de l'appel d'offres peut proposer à Total d'intégrer le projet à condition qu'il accepte de combiner sa technologie avec celle du concurrent.

Ce comportement de coopération entre concurrents est accentué par le comportement des Etats. Au regard de l'augmentation de la demande pour les appels d'offres, les Etats prennent conscience de l'avantage qu'ils ont en détenant l'accès aux zones géographiques. Ils en

\footnotetext{
${ }^{8}$ Article de presse Capital (2016), Eric Wattez. (2016, Mars 7), « 50 milliards engloutis, zéro baril de pétrole, le désastre du gisement de Kashagan ».
} 
viennent même à modifier les contrats pour ne plus supporter le risque de l'échec des projets. A la suite de ce changement de contrat, les projets d'E\&P deviennent instantanément plus risqués pour les entreprises puisque le risque d'échec n'est plus partagé avec l'Etat hôte. L'augmentation du niveau de risque favorise aussi le recours aux technologies des concurrents pour manager le risque.

\section{La cocréation de nouvelles technologies avec un concurrent (1970 - à aujourd'hui)}

A partir des années 1970, la stratégie poursuivie par Total et les quatre autres grands groupes pétroliers consistent à se spécialiser sur un nouveau marché : le marché de l'E\&P de pétrole difficilement accessible (e.g., l'exploration en haute mer). Cette stratégie est rendue nécessaire par l'ouverture et l'intensification de la compétition pour les zones géographiques contenant potentiellement du pétrole. Les entreprises nationales de pétrole commencent à maîtriser les technologies nécessaires à l'exploitation des gisements et évincent progressivement les majors des gisements les plus rentables. Total craint que ces entreprises nationales de pétrole s'intéressent de plus en plus à l'exploitation de gisement en dehors de leurs territoires, et viennent les concurrencer directement dans les appels d'offres. Entre 2004 et 2013, la production des cinq majors, Exxon Mobil, Shell, BP, Chevron et Total a diminué de 25\% (Greggio, Mafféï, 2015).

Total et les grands groupes des gisements doivent donc réussir à se différencier de ces entreprises nationales qui les rattrapent au niveau technologique. Un des moyens est de continuer les stratégies de combinaison des technologies entre majors. En combinant leurs dernières technologies stratégiques, l'efficience technologique reste un avantage concurrentiel. Une autre stratégie mise en place par Total et les autres majors consiste à se spécialiser dans des zones difficiles d'accès, c'est-à-dire des zones qui n'ont jamais été explorées à cause de la complexité de l'exploration, et de l'absence de technologie permettant de dépasser cette complexité (exemple : l'exploration en mer très profonde). Ainsi, l'engagement dans ces zones difficiles d'accès les protègent des entreprises nationales qui n'ont ni la R\&D, ni l'envie de gérer ces projets complexes. Mais l'enjeu est important et les coûts de ces projets sont colossaux et surtout marqués par l'incertitude de réussir à développer la technologie adéquate pour gérer ces contextes difficiles.

Cette stratégie est renforcée par une baisse généralisée de la production du pétrole. Pour faire face à cette baisse, les Etats vont chercher à exploiter de plus en plus de zones géographiques difficiles d'accès. Pour ces territoires, seuls les cinq majors ont les compétences et la capacité de développer une technologie spécifique permettant de surmonter l'accès difficile. Cependant, quelle que soit l'issue de l'appel d'offres, les majors ne s'y engagent pas seuls. Ces projets sont de facto plus complexes, plus coûteux, plus longs et plus intensifs en technologies nouvelles que les projets existants (e.g. 20 fois plus coûteux, non finançable sur fonds propres, avec une forte probabilité d'échec, d'une durée d'immobilisation de l'investissement d'une vingtaine 20 années). Un haut dirigeant de Total interviewé en 2014 met en avant le nécessité de coopérer, en particulier lorsqu'il s'agit de projets en mer profonde: "C'est très lourd à supporter cette phase d'exploration donc on y va à plusieurs. Et ce qui est encore plus lourd c'est la partie d'appréciation et surtout de développement. Il s'agit de développer en mer profonde le gaz de pétrole. Là, la mise de fond est énorme : on ne peut pas le faire seul. »Un autre haut dirigeant confirme que le recours à un concurrent permet de diversifier le risque : "L'idée, c'est quand même de réduire les risques ou d'étaler nos participations dans des entreprises sur davantage d'entreprises, et puis de gérer un portefeuille de positions de façon plus équilibrée, pour que ce qui va mal se compense par ce qui va bien. » Total ne peut donc pas entreprendre ces projets seule. Or, les banques refusent 
de faire des emprunts spécifiques pour ces projets caractérisés par deux tiers d'échecs ${ }^{9}$. Total est donc contraint de travailler avec ses concurrents pour cofinancer les projets et partager les risques. Les concurrents sont les seuls partenaires qui souhaitent et veulent s'engager dans ces projets.

De plus, cette coopération est stratégique car elle permet d'optimiser toutes les décisions et surtout de développer une nouvelle technologie nécessaire à l'aboutissement du projet. L'idée est de créer une structure où l'entreprise qui opère l'exploration et la production est composée des meilleurs experts, quelle que soit leur entreprise d'origine (cf. figure 6). Cette structure fait en sorte de diversifier les idées en confrontant celles de leurs experts à celles d'autres experts dans le même domaine mais provenant d'une autre entreprise. Un des hauts dirigeants met ainsi en avant l'intérêt d'être challenger : "quand on n'a que l'avis de ses experts à soi, on a qu'un seul son de cloche. Quand on est en JV[Joint-Venture], il y a une discussion, et souvent, ce qui a été dit par trois compagnies, à la fin des fins, c'est plus intelligent même si la discussion est animée et qu'on hurle et que l'on n'est pas content des partenaires. Le management n'est pas fâché des fois de se dire qu'il y a l'avis de CHEVRON, en plus de l'avis de TOTAL, et puis l'avis de CONOCO en plus de l'avis de CHEVRON, et que ça permet de relativiser des choses, on n'a pas tous les mêmes idées, on se fait critiquer [...], c'est quand même un truc qui est très civilisé où tout le monde est gagnant. La discussion doit amener les choses plus loin... ». Cette organisation a démontré qu'elle pouvait conduire à la création de nouvelles technologies permettant d'explorer et de produire dans des zones difficiles d'accès. Par exemple, Total avait une technologie efficace de conceptualisation et d'évaluation des potentiels de pétrole et de gaz, mais il ne pouvait pas utiliser cette technologie pour le gaz de schiste. En partageant sa technologie avec un concurrent maîtrisant les technologies actuelles d'évaluation des potentiels de gaz de schistes, ils ont pu ensemble cocréer une nouvelle technologie spécifique pour l'évaluation et la conceptualisation du gaz de schiste.

\footnotetext{
${ }^{9}$ Rapport de l'opecst, Sénat, 9 avril 2014.
} 
Figure 6 - Coopération technologique entre concurrents (cocréent une nouvelle technologie)

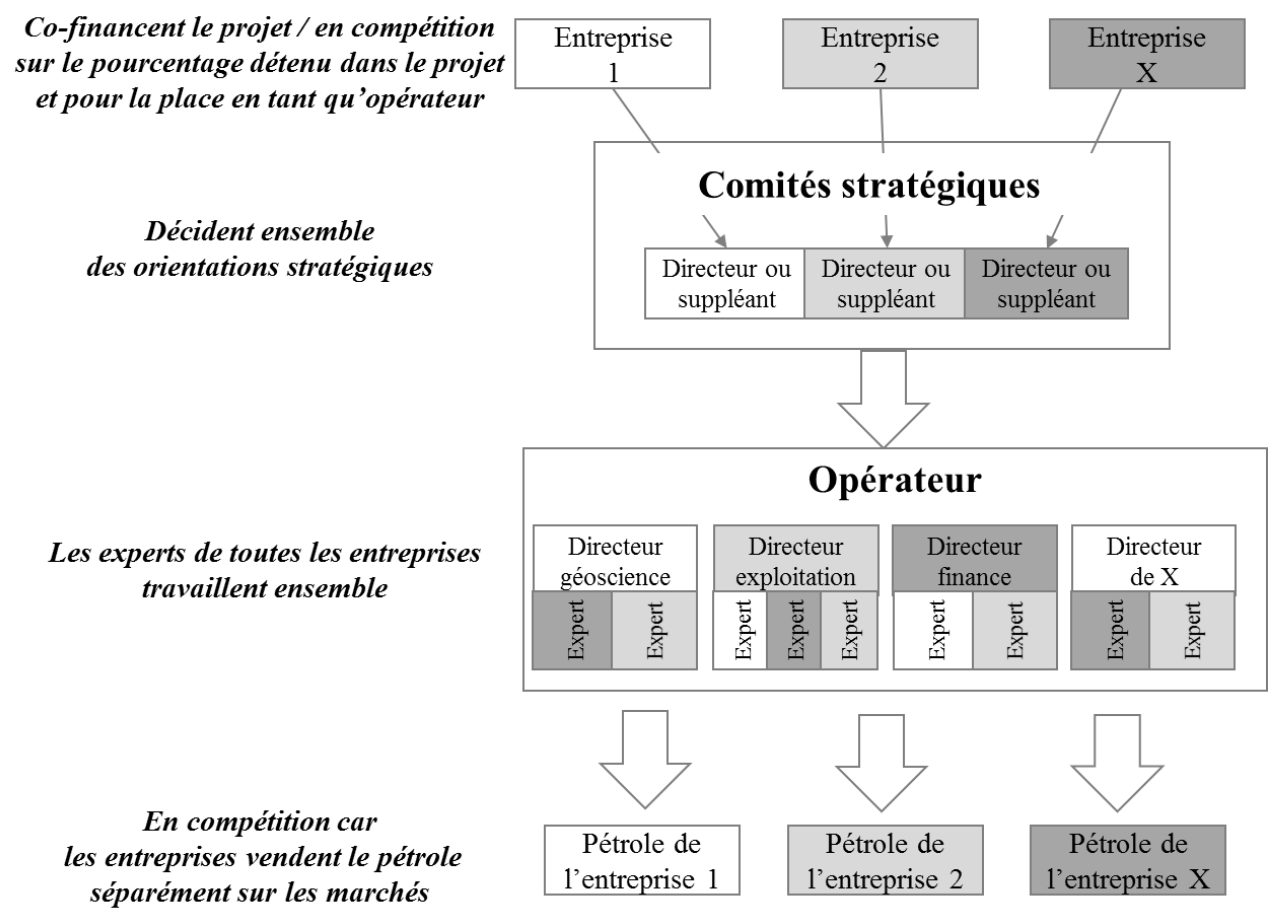

Source : auteur

\section{Synthèse du cas}

Total est bien en compétition sur la technologie avec les autres grands groupes comme Exxon Mobil, Shell, Chevron, BP ou ConocoPhillip. Mais malgré cette forte compétition, ils coopèrent pour l'accès aux zones géographiques à explorer et pour le développement d'une technologie efficiente qui permettra d'optimiser l'exploration et la production. La coopération pour le développement d'une technologie efficiente prend deux formes différentes (cf. tableau 3) : soit ils combinent leurs technologies sans les partager (ils se répartissent les tâches en fonction des technologies qu'ils possèdent et sur la base de cette répartition, chacun effectue ses tâches en interne), soit ils cocréent une nouvelle technologie qui n'existait pas avant en partageant leurs technologies (recherche ensemble à créer une nouvelle technologie). Ces choix de partager ou non la technologie repose sur des déterminants internes et externes différents. 
Tableau 3 - Gestion des technologies dans les projets d'E\&P de Total

\begin{tabular}{|c|c|c|}
\hline & Avant les années 70 & Après les années 70 \\
\hline $\begin{array}{l}\text { Pourquoi } \\
\text { développer } \\
\text { une } \\
\text { technologie } \\
\text { avec un } \\
\text { concurrent } \\
\text { direct? }\end{array}$ & $\begin{array}{l}\text { Accéder aux zones } \\
\text { géographiques avec un fort } \\
\text { potentiel de pétrole } \\
\text { (gagner l'appel d'offres) } \\
\text { Accéder à la technologie } \\
\text { du concurrent qui sur } \\
\text { certaines tâches est } \\
\text { meilleure (réduit le coût et } \\
\text { le risque du projet) }\end{array}$ & $\begin{array}{l}\text { Explorer et produire dans les } \\
\text { zones géographiques } \\
\text { difficiles d'accès } \\
\text { Manager l'incertitude de } \\
\text { pouvoir explorer et produire } \\
\text { dans cette zone } \\
\text { Développer un avantage } \\
\text { concurrentiel qui les } \\
\text { différencie des imitateurs } \\
\text { (sociétés nationales de } \\
\text { pétrole) }\end{array}$ \\
\hline $\begin{array}{l}\text { Modalités de } \\
\text { gestion des } \\
\text { technologies }\end{array}$ & $\begin{array}{c}\text { Combinent leurs technologies sans } \\
\text { les partager } \\
\text { (Ils se répartissent les tâches en } \\
\text { fonction des technologies qu'ils } \\
\text { possèdent et sur la base de cette } \\
\text { répartition, chacun effectue ses } \\
\text { tâches en interne) }\end{array}$ & $\begin{array}{c}\text { Co-créent une nouvelle technologie } \\
\text { qui n'existait pas avant en partageant } \\
\text { leurs technologies } \\
\text { (Ils créent une Joint Ventureet } \\
\text { recherchent ensemble à créer une } \\
\text { nouvelle technologie) }\end{array}$ \\
\hline $\begin{array}{l}\text { Exemple de } \\
\text { technologies }\end{array}$ & $\begin{array}{l}\text { Un ordinateur très puissant ou un } \\
\text { outil de désulfuration du gaz }\end{array}$ & $\begin{array}{l}\text { Les techniques de modélisation de } \\
\text { présence de pétrole }\end{array}$ \\
\hline $\begin{array}{l}\text { Eléments } \\
\text { externes } \\
\text { déclencheurs }\end{array}$ & $\begin{array}{l}\text { Intensification de la } \\
\text { concurrence } \\
\text { Coût de la R\&D } \\
\text { Niveau de risque des } \\
\text { projets }\end{array}$ & $\begin{array}{l}\text { Demande des Etats d'avoir } \\
\text { plus de zones d'exploration et } \\
\text { de production } \\
\text { Absence de technologie sur le } \\
\text { marché capable de répondre } \\
\text { aux attentes de l'Etat }\end{array}$ \\
\hline $\begin{array}{c}\text { Eléments } \\
\text { internes } \\
\text { déclencheurs }\end{array}$ & $\begin{array}{l}\text { Possède une technologie } \\
\text { qui intéresse les } \\
\text { concurrents } \\
\text { Technologies internes } \\
\text { insuffisantes pour gagner } \\
\text { seul les appels d'offres }\end{array}$ & $\begin{array}{l}\text { Vulnérabilité face aux } \\
\text { nouveaux entrants } \\
\text { Fonds propres insuffisants et } \\
\text { incertitude sur } \\
\text { l'aboutissement du projet } \\
\text { Besoin de se faire challenger, } \\
\text { et de combiner ses } \\
\text { technologies avec celles des } \\
\text { concurrents pour développer } \\
\text { la technologie recherchée }\end{array}$ \\
\hline
\end{tabular}

Source : auteur

\section{DISCUSSION ET CONCLUSION}

\section{Le management de la coopétition : partager ou ne pas partager ?}

Premièrement, elle enrichit notre compréhension des pratiques de management de la coopétition, en confirmant l'existence de deux managements différents en matière de partage 
de technologie (i.e., deux managements reposant sur deux structures de projets différentes : l'équipe projet séparée et l'équipe projet coopétitive). Nous confirmons que l'une n'est pas supérieure à l'autre mais que les deux sont utilisées en fonction du contexte et de l'objectif de la relation de coopétition. Il y a donc un réel intérêt à approfondir les recherches du management de la coopétition dans deux directions différentes.

Notre étude de cas le confirme. La première direction consiste à explorer les modalités pour atteindre l'objectif commun coopétitif sans partager la technologie (e.g., Pellegrin-Boucher, Fenneteau, 2007 ; Ritala, Hurmelinna-Laukkanen, 2009 ; Yami, Nemeh, 2014). La deuxième direction cherche à comprendre comment partager et simultanément minimiser les effets négatifs de ce partage (Enberg, 2012 ; Fernandez, Chiambaretto, 2016 ; Le Roy, Fernandez, 2015). En effet, notre cas longitudinal de Total confirme empiriquement les deux pratiques de gestion de coopétition et, surtout, met en avant l'absence de supériorité d'une pratique sur l'autre. Nous confirmons leurs existences empiriques en retrouvant au sein de Total les deux structures de projets récemment identifiées par Fernandez et al. (2017) : l'équipe projet séparée et l'équipe projet coopétitive. Parfois Total et ses concurrents combinent les technologies sans les partager et parfois, ils partagent et recombinent leurs technologies. Plus que tout, notre cas corrobore la pertinence des deux pratiques et donc, des deux directions de la littérature sur le management de la coopétition, en mettant en avant l'absence de supériorité d'une pratique sur l'autre. En effet, Total a continué à garder des structures projet en « équipe projet séparée » alors qu'il avait expérimenté avec succès l' «équipe projet coopétitive ».

\section{Les déterminants des pratiques de management de la coopétition}

Si la première pratique, l'équipe projet séparée, est intuitive car elle permet de bénéficier des avantages de la coopétition sans supporter le risque de l'internalisation, la deuxième ne l'est pas moins. En effet, l'entreprise supportera un risque d'internalisation des technologies bien plus élevé. C'est pourquoi notre recherche a cherché à comprendre pourquoi Total a parfois eu besoin de passer d'une pratique relativement plus sûre à une pratique plus risquée en matière de partage de connaissances. Notre recherche a identifié plusieurs déterminants multiniveaux (cf. figure 7).

Le premier niveau identifié est lié aux caractéristiques de l'environnement. L'entreprise évolue dans une industrie avec des menaces et des opportunités différentes. Notre étude de cas révèle que trois variables ont impacté le choix de Total de passer d'une combinaison de technologies entre concurrents sans partager, à un partage de ses technologies avec ses concurrents : (1) l'augmentation de l'intensité concurrentielle (i.e., l'arrivée de nouveaux concurrents), (2) le niveau de risque et le coût des projets (i.e., un projet d'exploration et production est déjà très coûteux et risqué, mais un projet similaire en mer profonde l'est d'autant plus), (3) la convergence technologique (i.e., les nouveaux concurrents y arrivent relativement aussi bien que les leaders du secteur). L'intensification de ces variables menace la survie de Total et des autres majors (e.g., perte sèche trop importante, différentiel technologique trop faible pour maintenir ses rentes). Ce contexte explique le choix pertinent de Total lorsqu'il partage ses technologies dans le but d'augmenter les chances de succès du projet.

Ainsi, lorsqu'une entreprise voit la valeur de sa technologie s'éroder avec le temps, et que de nouveaux entrants réduisent progressivement l'écart technologique, ce n'est qu'une histoire de temps avant que ces nouveaux entrants deviennent de véritables rivaux tout aussi armés technologiquement. Le partage de technologie devient alors une solution pour renouveler son avantage concurrentiel. Même si aucune généralisation de type statistique n'est possible à partir d'un cas unique, le partage de technologie dans les stratégies de coopétition technologique peut être un moyen de créer une capacité dynamique au sens de Teece (2014). 
Inversement, la coopétition technologique sans partage de technologie serait une capacité ordinaire permettant l'efficience technologique mais pas un renouvellement de l'avantage concurrentiel. Nos analyses ouvrent donc tout un champ de recherches sur les capacités dynamiques offertes par la coopétition technologique. Elles identifient aussi de nouveaux outils pour les leaders des industries de plus en plus concurrencées par les géants du web (e.g., Google, Amazon). En effet, dans l'industrie pharmaceutique, pour faire face à l'arrivée de Google, les leaders pourraient coopérer et partager leurs technologies afin de cocréer ensemble un nouvel avantage concurrentiel.

Le deuxième niveau concerne la relation et la capacité des autres entreprises partenaires à apporter de la valeur à la technologie partagée. En effet, si l'entreprise partenaire ne peut pas apporter de valeur, il est inutile de partager la technologie : cela constituerait une prise de risque inutile. Dans ce contexte particulier, l'entreprise a intérêt à favoriser la combinaison de technologies sans partage. Par exemple, Total n'a pas eu intérêt à partager son ordinateur très puissant (i.e., un des ordinateurs les plus puissants du monde). Il a pu faire les calculs en interne et ne partager que les résultats. Par contre, lorsque la technologie possédée doit être améliorée pour répondre à des contextes spécifiques, le concurrent peut s'avérer être un partenaire pertinent. Effectivement, plus les entreprises sont des concurrents directs, plus les technologies sont simultanément similaires et complémentaires, ce qui rend le partenaire apte à challenger les idées et les technologies partagées. Ainsi, en acceptant de partager sa technologie avec un concurrent spécialiste du gaz de schiste, Total a pu utiliser sa technologie de modélisation des sols pour l'identification de la présence des gaz de schiste. Cette idée, que tous les concurrents n'ont potentiellement pas le même effet de levier sur les ressources d'une entreprise, a été très peu étudiée dans la littérature sur la coopétition. Dans celle-ci, les études ont tendance à majoritairement comparer les concurrents aux non concurrents, sans faire de distinction entre les différents concurrents (e.g., Hamouti et al., 2014; Le Roy et al., 2016). L'étude de cas révèle un besoin de complexifier nos connaissances sur la coopétition en distinguant les types de concurrents.

Le troisième niveau nous amène à l'intention stratégique. Lorsque l'objectif est uniquement de combiner les technologies pour améliorer l'efficience technologique du projet, le partage de technologies est inutile et risqué. Par contre, pour créer une technologie totalement nouvelle, les coopétiteurs ont intérêt à s'engager dans une interaction intense et ouverte. Ce niveau confirme les travaux de Fernandez et al. (2017) soutenant la pertinence des structures de projet de type « équipe projet coopétitive » pour l'innovation radicale, et les structures de projet de type « équipe projet séparée » pour les projets d'innovation incrémentale.

Le dernier niveau concerne les caractéristiques des acteurs. Nous avons identifié deux variables dans ce niveau: la perception d'une vulnérabilité technologique et la capacité d'intégration du paradoxe par les individus. La première est directement reliée au niveau « environnement». L'environnement rend les technologies actuelles de l'entreprise plus ou moins vulnérables. L'entreprise n'acceptera de les partager pour les renouveler que si, et seulement si, elle ressent cette vulnérabilité et si elle considère qu'un sacrifice est l'unique moyen de la renouveler. Mais pour être capable de faire ce « sacrifice », il faut avoir intégré le paradoxe de la coopétition. La littérature antérieure avait identifié que tous les individus ne sont peut-être pas capables d'intégrer le paradoxe et de dépasser la relation de marché uniquement compétitive pour y intégrer de la coopération (Bengtsson, Kock, 2000; Walley, 2007). Ce besoin de sacrifier la technologie pour la renouveler n'est peut-être pas perceptible par tous les individus. Il serait intéressant de retourner étudier Total afin d'identifier s'il y a eu des réticences au niveau des individus de passer d'une équipe projet séparée à une équipe projet coopétitive. En tout cas, nous pouvons faire le constat que les deux pratiques basées sur le partage ou le non partage de la technologie n'implique pas le même besoin d'intégration. Pour les projets coopétitifs sans partage, les individus n'ont pas besoin d'intégrer le 
paradoxe : cette stratégie de coopétition serait donc accessible à tous les individus. Par contre, pour les projets coopétitifs avec partage, les individus ont besoin d'intégrer le paradoxe : cette stratégie serait donc réservée à certains individus capables d'intégrer qu'ils ne sont pas uniquement dans une relation de compétition avec le concurrent mais bien une relation simultanément de compétition et de coopération.

Figure 7 - Modèle conceptuel des déterminants du partage ou de l'absence de partage de technologie dans une stratégie de coopétition technologique

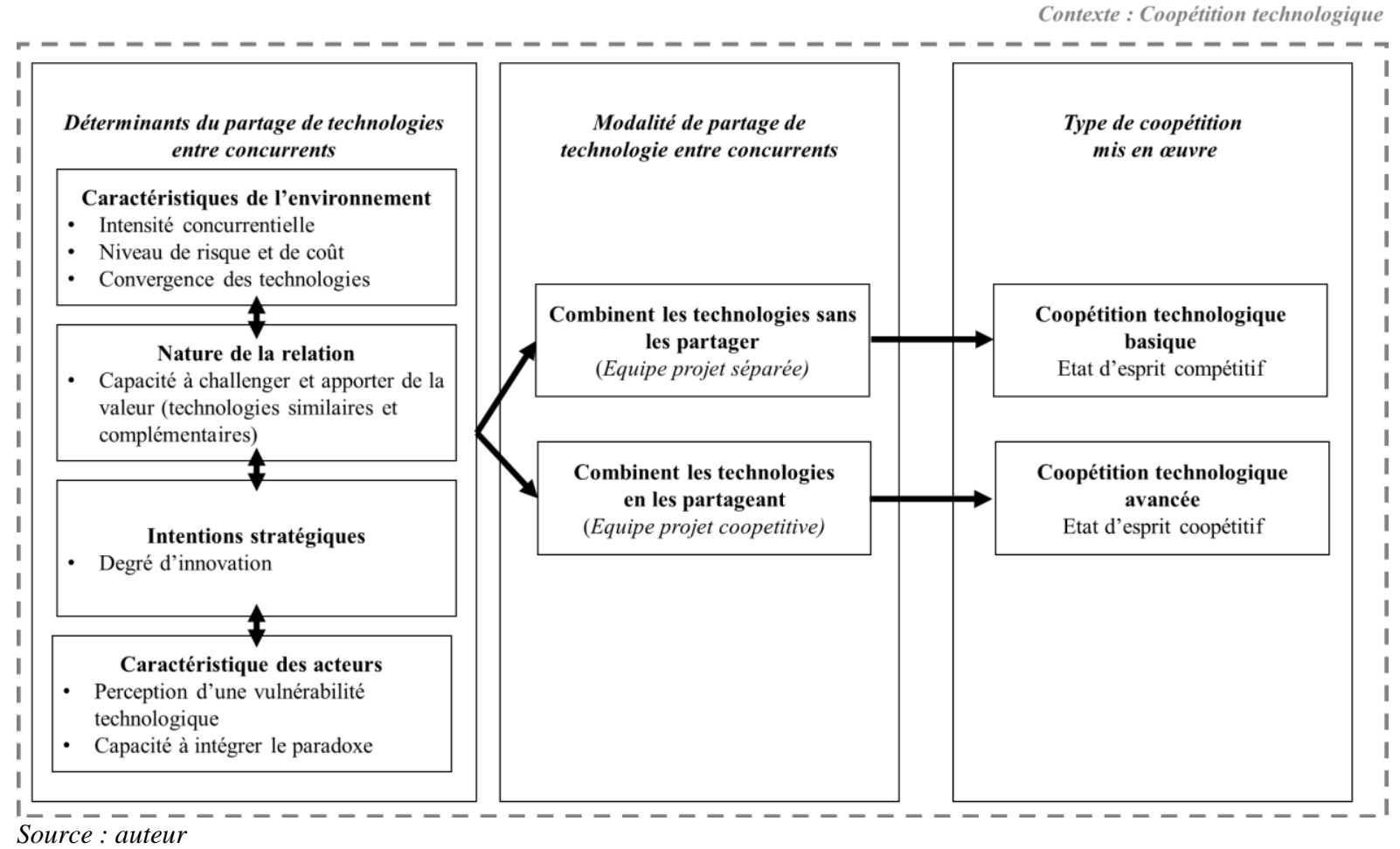

\section{Vers une nouvelle typologie de la coopétition : coopétition basique versus avancée}

Nous contribuons à la littérature sur la coopétition en mettant en avant la coexistence académique et empirique de deux pratiques de management de la coopétition technologique. Chaque pratique est basée sur un comportement différent de partage de technologie. Plus précisément, nous complexifions notre compréhension de la coopétition en identifiant une nouvelle typologie basée sur le comportement de partage de technologies. En effet, le nombre croissant d'articles sur la coopétition a mis en avant que la coopétition pouvait être horizontale ou verticale (Chiambaretto, Dumez, 2016), entre un acteur ou plusieurs (Dagnino, Padula, 2002; Gnyawali et al., 2008), et impliquer différentes activités comme la coopétition pour la R\&D ou la coopétition pour la vente (Pellegrin-Boucher, Le Roy, à paraître). Notre cas questionne la pertinence de ces typologies pour expliquer le partage ou le non partage de technologies dans les stratégies de coopétition. En effet, le cas de Total montre qu'à l'inverse de Oxley et Sampson (2004), Total coopère avec ses concurrents de façon étendue : la coopération et le partage de technologie dépasse les activités de pure R\&D et concerne aussi le projets d'E\&P. De manière identique, le cas de Total montre la non pertinence d'utiliser la distinction coopétition verticale ou horizontale pour expliquer si une entreprise partage ou non ses technologies. Théoriquement, lorsque le partenaire est un concurrent direct, l'entreprise ne devrait pas partager ses technologies (Pellegrin-Boucher et al., 2013). Notre cas Total montre 
une situtation où une entreprise peut avoir un intérêt à partager avec son concurrent direct. Concernant la typologie sur le nombre d'acteurs, notre cas montre aussi qu'il est possible de coopérer et de partager sa technologie avec un nombre important de concurrents. Dans le projet d'E\&P Kashasgan, Total coopère et partage sa technologie avec l'américain Exxon Mobil, l'anglo-néerlandais Shell, l'italien ENI, le chinois CNPC et le japonais Impex.

Notre cas suggère l'existence d'une autre typologie directement basée sur le partage de technologies (cf. tableau 4). Il y aurait la coopétition basique qui consiterait à coopérer sans partager de technologies pour atteindre l'efficience technologique. Et, il existerait la coopétition avancée qui consisterait à coopérer en partageant ses technologies pour permettre la création d'une nouvelle technologie répondant à des besoins de renouvellement de l'avantage concurrentiel. Cette nouvelle typologie a un double intérêt empirique et académique. L'intérêt empirique de cette distinction est qu'elle met en avant, dans un premier temps, une coopétition facile d'accès dans laquelle les managers peuvent s'engager et garder leur esprit compétitif. Puis, dans un second temps, cette distinction souligne l'existence d'une coopétition avancée dans laquelle les managers sont obligés de partager de manière intensive leurs technologies et donc d'intégrer le paradoxe de la coopétition. Au niveau académique, notre recherche met en avant des opportunités et des enjeux radicalement différents entre les deux types de coopétition (e.g., création d'une nouvelle technologie versus efficience technologique ; risque de fuite de technologie versus pas de fuite possible ; besoin d'intégrer le paradoxe versus absence d'intégration du paradoxe).

Notre recherche met en avant un réel besoin de distinguer les recherches actuelles en fonction de ces deux types de coopétition. Cependant nos contributions sont limitées par l'industrie très spécifique dans laquelle a été menée notre étude de cas. Il est donc nécessaire d'approfondir nos connaissances en répliquant cette recherche dans d'autres entreprises et d'autres industries. L'objectif de ces futures recherches pourraient être d'affiner notre compréhension de ces deux types de coopétition, pour ensuite étudier leur co-existence. Dans le champ de la coopétiton, nous sommes au début d'une recherche nécessitant de plus en plus de rigueur sur la description du partage ou non de technologies, et amenant ainsi à de plus en plus de complexité à la stratégie de coopétition.

Tableau 4 - Différence entre coopétitions basique et avancée

\begin{tabular}{ccc}
\hline But & Coopétition basique & Coopétition avancée \\
\hline $\begin{array}{c}\text { Risque que le concurrent } \\
\text { internalise les } \\
\text { connaissances }\end{array}$ & $\begin{array}{c}\text { Efficience technologique } \\
\text { combinent des technologies } \\
\text { existantes })\end{array}$ & $\begin{array}{c}\text { Cocréation d'une technologie } \\
\text { (créent une nouvelle } \\
\text { technologie) }\end{array}$ \\
\hline $\begin{array}{c}\text { Besoin d'un principe } \\
\text { d'intégration au niveau } \\
\text { des individus }\end{array}$ & Faible & Fort \\
\hline Structure projet & Non & Oui \\
\hline $\begin{array}{c}\text { Type de technologie } \\
\text { partagée }\end{array}$ & $\begin{array}{c}\text { Technologie non spécifique } \\
\text { (réutilisable telle quelle dans } \\
\text { un autre projet) }\end{array}$ & $\begin{array}{c}\text { Technologie spécifique } \\
\text { (besoin de l'adapter en } \\
\text { fonction des projets) }\end{array}$ \\
\hline
\end{tabular}

Source : auteur

\section{BIBLIOGRAPHIE}

ARRANZ, N., DE ARROYABE, J. (2008), The choice of partners in R\&D cooperation: An 
empirical analysis of Spanish firms, Technovation, 28(1-2), 88-100.

BAUMARD, P. (2009), An asymmetric perspective on coopetitive strategies, International Journal of Entrepreneurship and Small Business, 8(1), 6.

BAUMARD, P. (2010), La logique de l'avantage coopétitif, In: Stratégies de coopétition: rivaliser et coopérer simultanément (DeBoeck, ed.), 81-100. Bruxelles, Belgique: Collection Méthodes et Recherches.

BENGTSSON, M., KOCK, S. (2000), "Coopetition" in business networks - to cooperate and compete simultaneously, Industrial Marketing Management, 29, 411-426.

BEZ, S., LE ROY, F., PELLEGRIN-BOUCHER, E. , GOURSAUD, P. (2014), Le patient anglais: lorsque l'alliance entre Sanofi et BMS donne naissance à une innovation médicale majeur, In: Coopétition: S'allier à ses concurrents pour gagner (P. Marquès, J. Granata, eds.), Pearson Fr., 125-154. Tours.

BOUNCKEN, R. (à paraître.) Devils' Dance? Symphony of Challenges and Merits for Coopetitive Innovation, In: Handbook of coopetition (F. Le Roy, W. Czakon, A.-S. Fernandez, P. Chiambaretto, eds.), forthcoming.

BOUNCKEN, R., FREDRICH, V. (2012), Coopetition: performance implications and management antecedents, International Journal of Innovation Management, 16(5), 1250028 1-1250028 28.

BOUNCKEN, R., KRAUS, S. (2013), Innovation in knowledge-intensive industries: The double-edged sword of coopetition, Journal of Business Research, 66(10), 2060-2070. Elsevier Inc.

BRANDENBURGER, A., NALEBUFF, B. (1996), Co-Opetition,. New York: Doubleday currency.

BROWNING, L., BEYER, J., SHETLER, J. (1995), Building Cooperation in a Competitive Industry: Sematech and the Semiconductor Industry., Academy of Management Journal, 38(1), 113-151.

CHIAMBARETTO, P., DUMEZ, H. (2016), Towards a typology of coopetition: a multilevel approach, International Studies of Management and Organization, 46(3), 110-129.

DAGNINO, G. , PADULA, G. (2002), Coopetition Strategy: a new kind of interfirm dynamics for value creation, EURAM - The European Academy of Management Second Annual Conference - 'Innovative Research in Management', May 9- 11, Stockholm.

DEPEYRE, C., DUMEZ, H. (2007), Le rôle du client dans les stratégies de coopétition, Revue française de gestion, 33(176), 99-110.

DORN, S., SCHWEIGER, B., ALBERS, S. (2016), Levels, phases and themes of coopetition: A systematic literature review and research agenda, European Management Journal, $34(5), 484-500$

DUMEZ, H. (2013), Méthodologie de la recherche qualitative: les 10 questions clés de la démarche compréhensive,. Vuibert.

DUMEZ, H. (2016), Comprehensive Research: a methodological and epistemological introduction to qualitative research,. Copenhagen: Copenhagen Business School Press.

DYER, J., SINGH, H. (1998), The relational view: cooperative strategy and sources of interorganizational competitive advantage, Academy of management review, 23(4), 660679.

EISENHARDT, K. (1989), Building theories from case study research, Academy of management review, 14(4), 532-550.

ENBERG, C. (2012), Enabling knowledge integration in coopetitive R\&D projects : The management of conflicting logics, International Journal of Project Management, 30(7), 771-780

ESTRADA, I., FAEMS, D., DE FARIA, P. (2016), Coopetition and product innovation performance: The role of internal knowledge sharing mechanisms and formal knowledge 
protection mechanisms, Industrial Marketing Management, 53, 56-65.

FAEMS, D., JANSSENS, M., VAN LOOY, B. (2010), Managing the Co-operationCompetition Dilemma in R\&D Alliances: A Multiple Case Study in the Advanced Materials Industry, Creativity and Innovation Management, 19(1), 3-22.

FERNANDEZ, A.-S., CHIAMBARETTO, P. (2016), Managing tensions related to information in coopetition, Industrial Marketing Management, 53, 66-76.

FERNANDEZ, A.-S., LE ROY, F. (2010), Pourquoi coopérer avec un concurrent? Une approche par la RBV, Revue française de gestion, 36(204), 155-169.

FERNANDEZ, A.-S., LE ROY, F. (2015), Les stratégies de coopétition comme source de tensions: le cas EADS-Thales dans l'industrie des satellites de télécommunications, Management International, 19(3), 115-128.

FERNANDEZ, A.-S., LE ROY, F., CHIAMBARETTO, P. (2017), Implementing the right project structure to achieve coopetitive innovation projects, Long Range Planning, online, 1-22.

FERNANDEZ, A.-S., LE ROY, F., GNYAWALI, D. (2014), Sources and management of tension in co-opetition case evidence from telecommunications satellites manufacturing in Europe, Industrial Marketing Management, 43(2), 222-235.

GNYAWALI,D. , CHARLETON, T. (2017), Divergence and convergence of coopetition research: bridging the conversations and shaping for research agenda, Strategic Management Society,. Houston.

GNYAWALI, D. , JUNYU, H., MADHAVAN, R. (2008), Co-opetition: promises and challenges, In: 21st Century Management: a Reference Handbook (C. Wankel, ed.), 386-398. London: Sage Publications.

GNYAWALI, D., MADHAVAN, R., HE, J., BENGTSSON, M. (2016), The competitioncooperation paradox in inter-firm relationships: A conceptual framework, Industrial Marketing Management, 53, 7-18.

GNYAWALI, D., PARK,B.-J. (2011), Co-opetition between giants: Collaboration with competitors for technological innovation, Research Policy, 40(5), 650-66.

GREGGIO, R., MAFFEÏ, B. (2015), Le «grand retour» des majors du pétrole à la faveur du troisième choc pétrolier-Classes d'entreprises et groupes stratégiques de l'industrie pétrolière, Gérer et comprendre, 120(Juin), 16-26.

HAMEL,G. (1991), Competition for competence and interpartner learning within international strategic alliances, Strategic Management Journal, 12(Special Issue:Global Strategy), 83-103.

HAMOUTI, R., ROBERT, F., LE ROY, F. (2014), Stratégie individuelle, stratégie de coopération verticale ou stratégie de coopétition. Quelle est la meilleure stratégie pour l'innovation produit?, Innovations, 43(1), 135-161.

LE ROY, F., CZAKON, W. (2016), Managing coopetition: the missing link between strategy and performance, Industrial Marketing Management, 53, 3-6.

LE ROY, F., ROBERT, M., LASCH, F. (2016), Choosing the best partner for product innovation: Talking to the enemy or to a friend ?, International Studies of Management Organization, 46(2-3), 136-158.

NIETO, M., SANTAMARÍA, L. (2007), The importance of diverse collaborative networks for the novelty of product innovation, Technovation, 27(6-7), 367-377.

OXLEY, J., SAMPSON, R. (2004), The Scope and Governance of International R\&D Alliances, Strategic Management Journal, 25(8/9), 723-749.

PARK, S., RUSSO, M. (1996), When competition eclipses cooperation: an event history analysis of joint venture failure, Management Science, 42(6), 875-890.

PELLEGRIN-BOUCHER, E., FENNETEAU, H. (2007), Le management de la coopétition: le cas du secteur des ERP, Revue française de gestion, 176, 111-134. 
PELLEGRIN-BOUCHER, E., LE ROY, F. (à paraître.) Managing selling coopetition : a case study of the ERP industry, European Management Review.

PELLEGRIN-BOUCHER, E., LE ROY, F., GURAU, C. (2013), Coopetitive strategies in the ICT sector: typology and stability, Technology Analysis, Strategic Management, 25(1), 71-89.

QUINTANA-GARCÍA, C., BENAVIDES-VELASCO, C. (2004), Cooperation, competition, and innovative capability: a panel data of European dedicated biotechnology firms, Technovation, 24(12), 927-938.

RITALA, P., HURMELINNA-LAUKKANEN, P. (2009), What's in it for me? Creating and appropriating value in innovation-related coopetition, Technovation, 29(12), 819-828.

RITALA, P., OLANDER, H., MICHAILOVA, S., HUSTED, K. (2015), Knowledge sharing, knowledge leaking and relative innovation performance: An empirical study, Technovation, 35, 22-31.

SANTAMARIA, L., SURROCA, J. (2011), Matching the goals and impacts of R\&D collaboration, European Management Review, 8(2), 95-109.

TEECE, D. (2014), The foundations of entreprise performance: dynamic and ordinary capabilities in an (economic) theory of firms, Academy of Management Perspectives, 28(4), 328-352.

WALLEY, K. (2007), Coopetition: An introduction to the subject and an agenda for research, International Studies of Management and Organization, 37(2), 11-31.

YAMI, S., NEMEH, A. (2014), Organizing coopetition for innovation: The case of wireless telecommunication sector in Europe, Industrial Marketing Management, 43(2), 250-260

YIN, R. (2014), Case study research: Design and methods, 5th ed. United States of America: Sage Publications. 